\title{
Long-term functional and quality of live assessment following post-traumatic distraction osteogenesis of the lower limb
}

\author{
Niels Willem Luitzen Schep • \\ Esther M. M. van Lieshout • Peter Patka • \\ Lucas M. M. Vogels
}

Received: 17 June 2009/ Accepted: 7 November 2009/Published online: 24 November 2009

(C) Springer-Verlag 2009

\begin{abstract}
Limb length discrepancy and segmental bone defects can be difficult problems to manage after fractures of the lower limb. Distraction osteogenesis can be applied to lengthen bone or to bridge intercalary defects by segmental bone transport. The purpose of this study was to assess the functional outcome and long-term quality of life after distraction osteogenesis of the lower limb when applied for post-traumatic problems. Three patients were treated with segmental transport for bone loss secondary to infection and debridement after a tibia fracture. Leg lengthening was performed in 12 patients with consolidated fractures (eight femurs and four tibias). Long-term functional outcomes were evaluated with the Short Form-36 Health Survey (SF-36) and the Lower Extremity Functional Scale (LEFS) in all patients. The mean follow-up was 9 years. Functional outcomes indicated moderate difficulty in patients after segmental transport and a little difficulty in patients after lengthening of consolidated fractures. The SF-36 scores varied two points for physical functioning and one point for bodily pain as compared to the SF-36 norm-based scores. In all other six domains, patient scores were comparable with the general population. In conclusion, the quality of life and functional outcome returned to normal after post-traumatic distraction osteogenesis of the lower limb.
\end{abstract}

N. W. L. Schep $(\varangle)$ · E. M. M. van Lieshout · P. Patka ·

L. M. M. Vogels

Department of Surgery-Traumatology, Erasmus MC,

University Medical Center Rotterdam, P.O. Box 2040,

3000 CA Rotterdam, The Netherlands

e-mail: n.schep@erasmusmc.nl
Keywords Lower limb - Distraction osteogenesis . Quality of life - SF-36 - LEFS

\section{Introduction}

Limb length discrepancy and segmental bone defects are difficult problems after fractures of the lower limb. The etiology of post-traumatic limb length discrepancy is malunion, axial collapse of comminuted fractures or through premature closure of the physis in children. A segmental bone defect may result from bone loss due to the trauma itself or due to debridement for osteomyelitis. Both situations may have a negative impact on the patient's quality of life and present as a complex challenge to treatment $[1,2]$.

Ilizarov pioneered treatment of these problems with a modular ring fixator and transosseous wires to stabilize the bone fragments. New bone was generated between osseous surfaces that were gradually pulled apart. This technique was named distraction osteogenesis [3]. Distraction osteogenesis allows the surgeon to perform limb lengthening for patients with post-traumatic leg length discrepancy or be used for bridging intercalary defects by segmental bone transport [3-6].

There are many reports of case series describing the surgical technique and complications around distraction osteogenesis. Some reports of functional outcome have been though using unvalidated scores with a short followup period. To our knowledge, the relation between posttraumatic distraction osteogenesis and quality of life has not been studied. The purpose of this work was to assess the functional outcome and long-term quality of life after distraction osteogenesis of the lower limb when applied in post-traumatic problems. 


\section{Materials and methods}

Fifteen adults were treated by distraction osteogenesis of the lower limb in the period 1997-2005. Three patients were treated with segmental transport for bone loss secondary to debridement for infection after a tibial fracture. Leg lengthening was performed in 12 patients with leg length inequality after fractures (eight femurs and four tibias). The demographic features of the patients are listed in Table 1.

With the use of an institutional review board-approved protocol, the medical records were reviewed, and patients were contacted to return for evaluation. A retrospective analysis was performed to determine the duration of treatment and healing index. The healing index is defined by the total duration of treatment divided by the number of centimeters of new bone formation (months/cm). Furthermore, complications such as pin-track infections and secondary operations were extracted from the medical records.

For all patients, functional outcomes were evaluated with two validated questionnaires: the Medical Outcomes Study Short Form-36 Health Survey (SF-36) and the Lower Extremity Functional Scale (LEFS) [7, 8]. All questionnaires were completed by the patients during their visit.

The SF-36 consists of multi-item scales that measure eight health domains: physical functioning (PF); role limitations due to physical health (RP); bodily pain (BP); social functioning (SF); vitality, energy, or fatigue (VT); general health perceptions $(\mathrm{GH})$; role limitations due to emotional problems (RE); general mental health $(\mathrm{MH})$. These are calculated to give a physical component score (PCS) and a mental component score (MCS). Scores ranging from 0 to 100 points are derived for each domain, with lower scores indicating poorer function. These scores are converted to a norm-based score and compared with the norms for the general population of the United States (1998). In the US population, each scale was scored to have the same average (50) and the same standard deviation (10 points). Calculating norm-based scores using the Dutch and US populations provides similar results for the eight health domains. However, the weighing factors for calculating PCS and MCS for the Dutch population were not available; therefore, the US population was used as reference. Consequently, on all scales, any score less than 50 falls below the general population mean.

The LEFS is a 20-item self-report measure of physical function. Each item is rated on a five-point scale (0-4), with lower scores representing greater difficulty. Total scores can range from 0 to 80 . Function is defined as follows: extreme difficulty or unable to perform activity (0-19 points), quite a bit of difficulty (20-39 points), moderate difficulty (40-59 points), a little bit of difficulty (60-79 points), and no difficulty (80 points).

Additionally, a standing full-length anteroposterior radiograph of the lower extremities was obtained. The mechanical axis deviation (MAD) was calculated in millimetres and defined as the distance from the center of the femoral condyles to the vertical line connecting the center of the femoral head to the center of the tibial plafond, the mechanical axis. Medial deviation of the mechanical axis was denoted as varus, whereas lateral deviation was denoted as valgus malalignment [9]. In all patients, postoperative limb length discrepancy was calculated as the difference between the lengths of both mechanical axes.

Table 1 Demographic Data

\begin{tabular}{lllll}
\hline Patient & $\begin{array}{l}\text { Age (year) at time } \\
\text { of accident }\end{array}$ & Gender & $\begin{array}{l}\text { Primary diagnosis, open fractures } \\
\text { have been classified according Gustilo }\end{array}$ & Lengthening procedure \\
\hline 1 & 25 & F & Closed tibia shaft fracture & Segmental bone transport tibia \\
2 & 25 & F & Grade II tibia shaft fracture & Segmental bone transport tibia \\
3 & 22 & M & Closed tibia shaft fracture & Segmental bone transport tibia \\
4 & 18 & F & Closed femur shaft and tibia shaft fracture & Primary lengthening femur \\
5 & 22 & M & Grade II femur shaft fracture & Primary lengthening femur \\
6 & 20 & M & Grade III tibia shaft fracture & Primary lengthening tibia \\
7 & 30 & M & Grade III tibia shaft fracture & Primary lengthening tibia \\
8 & 33 & M & Grade III femur shaft fracture and closed tibia shaft fracture & Primary lengthening femur \\
9 & 34 & M & Grade I femur shaft fracture & Primary lengthening femur \\
10 & 18 & M & Grade II tibia shaft fracture & Primary lengthening tibia \\
11 & 18 & M & Grade II femur shaft fracture & Primary lengthening femur \\
12 & 41 & M & Grade III femur shaft fracture & Primary lengthening femur \\
13 & 51 & M & Closed femur shaft fracture & Primary lengthening femur \\
14 & 35 & F & Closed tibia plateau fracture & Primary lengthening tibia \\
15 & 22 & M & Grade III femur shaft and closed tibia shaft fracture & Primary lengthening femur \\
\hline
\end{tabular}




\section{Operative technique}

Segmental bone transport was performed for bone loss secondary to debridement for infection. Once the infection had subsided, a monolateral external fixator for lengthening (Orthofix SRL, Verona, Italy) was positioned over the defect. A single longitudinal periosteal incision was made over the inferior third of the tibial tuberosity for metaphyseal lengthening. The cortex was circumferentially predrilled with a 3.2 drill. Then, a subperiosteal corticotomy was performed by connecting the drill holes with an osteotome (Fig. 1). Special care was taken to avoid periosteal damage. After a latency period of 7 days, the bone segment was distracted with a distraction rate of $1 \mathrm{ml}$, per day in four $0.25 \mathrm{~mm}$ increments. On reaching the docking site, the transported bone segment was fixed with a plate to the distal bone segment and autogenous bone graft added (Fig. 2).

Tibial lengthening for consolidated fractures was performed with a similar technique to that described earlier. In addition, a fibula osteotomy was performed at the junction of the distal and middle thirds of the diaphysis. For femoral lengthening, a subperiosteal corticotomy was carried out in the proximal metaphysis, corresponding with the subtrochanteric area. When lengthening was completed, the patients were encouraged to bear $100 \%$ of their weight after dynamization of the fixator.

\section{Results}

\section{Bone transport}

The three patients who underwent segmental transport of the tibia had a mean age of 24 years (range 22-25 years).

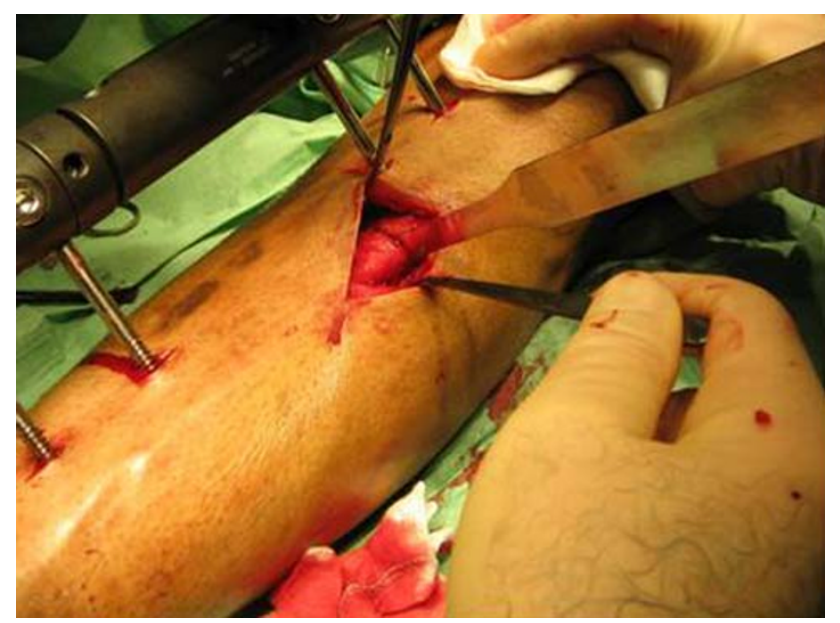

Fig. 1 A subperiosteal corticotomy was performed by connecting the drill holes with an osteotome
The average amount of lengthening in these three patients was $12 \pm 5 \mathrm{~mm}(\mathrm{SD})$. The fixators were in place for an average of $14 \pm 4$ months (range 9.9-16.8 months). The mean healing index was $1.4 \pm 0.9$ months $/ \mathrm{cm}$. One patient developed a pin tract infection, which was treated with oral antibiotics. Following segmental transport, all patients were treated with a plate fixation and autogenous bone grafting to allow consolidation at the docking site. One patient was treated with an additional corrective osteotomy of the tibia due to varus angulation of the tibia. After a mean follow-up of $6 \pm 3$ years, an average LEFS score of $47 \pm 12$ was found.

\section{Lengthening}

Leg lengthening was performed in twelve patients: eight femurs and four tibias. The median age was 26 years (range 18-51 years) in two female and ten male patients. The mean amount of lengthening was $6 \pm 4 \mathrm{~cm}$ for femoral and $4 \pm 2 \mathrm{~cm}$ for tibial distraction osteogenesis. The fixators were in place for an average of $8 \pm$ 5 months for femoral and $5 \pm 1$ for tibial lengthening. The mean healing index was $1.5 \pm 1.0$ months $/ \mathrm{cm}$ in the femur and $1.6 \pm 0.6 \mathrm{month} / \mathrm{cm}$ in the tibia. Five patients developed a pin tract infection in the femur and two patients in the tibia, of which two infections in the femur and one in the tibia were treated with intravenous antibiotics. The other four patients were treated for persisting fistula with operative debridement of the pin track after the fixator was removed. Osteoclasis for premature consolidation was performed in one patient during femoral distraction osteogenesis. One patient in the tibial distraction osteogenesis group underwent an ankle arthrodesis due to intractable pain in the ankle joint. The mean follow-up was $9.6 \pm 3.3$ years in the femoral and $10.5 \pm 3.9$ years in the tibial lengthening group. The average LEFS score was $61 \pm 13$ points after femoral and $62 \pm 18$ points after tibial lengthening.

The median SF-36 scores for each of the eight health domains were as follows: PF (48 \pm 11$), \operatorname{RP}(56 \pm 10)$, BP (49 \pm 10$), \mathrm{GH}(55 \pm 7)$, VT $(57 \pm 7), \mathrm{SF}(57 \pm 6), \mathrm{RE}$ $(56 \pm 4), \mathrm{MH}(55 \pm 5)$. The combined physical and mental component scores were $49 \pm 11$ and $57 \pm 6$, respectively. The median SF-36 scores for the 15 patients in all the eight domains are displayed in Fig. 3 for comparison with US population norms.

The mean MAD was $21 \pm 20 \mathrm{~mm}$ (range 0-59 mm) with five patients showing varus and eight patients showing valgus malalignment. One patient demonstrated no mechanical axis deviation, and in one patient no radiographs were taken due to pregnancy. The overall postoperative limb length discrepancy, defined as the difference in lengths of both mechanical axes, was $1.6 \pm 1.6 \mathrm{~cm}$. 
Fig. 2 The bone segment was distracted with a distraction rate of $1 \mathrm{~mm}$ per day. As soon as the bone segment had reached the docking site, a plate fixation with autogenous bone grafting was performed
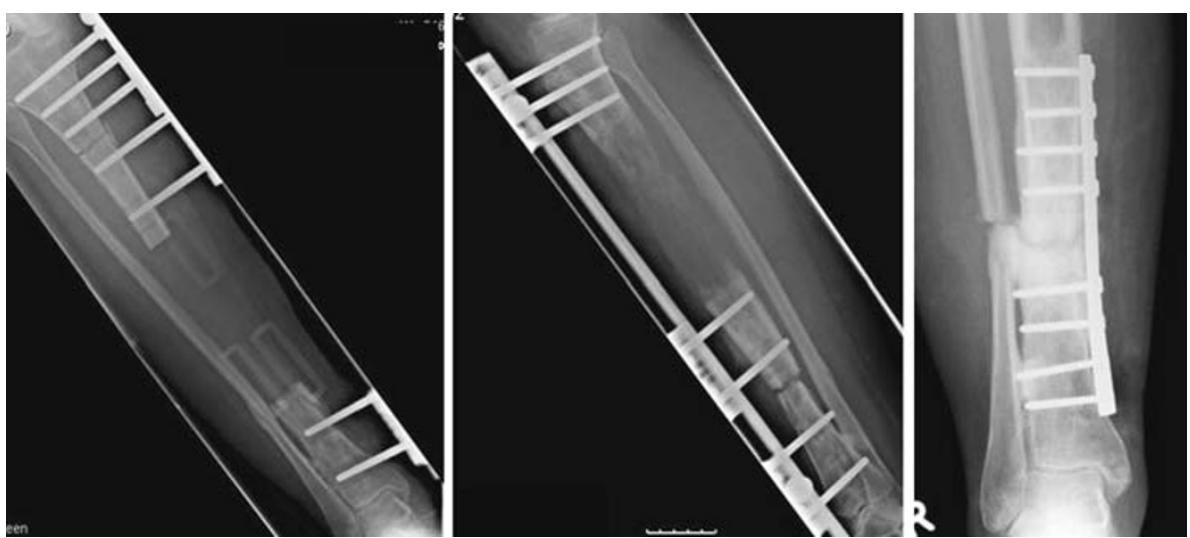

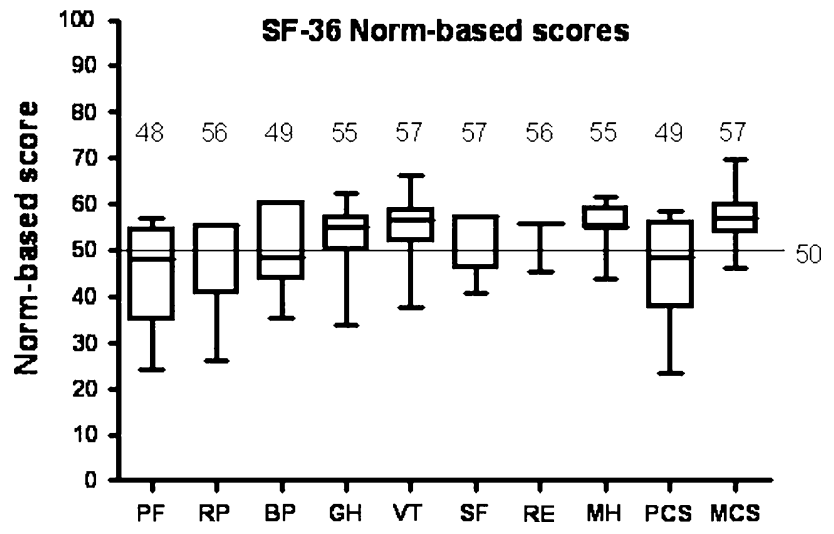

Fig. 3 Median SF-36 scores leg lengthening in comparison with US population norms. Physical functioning (PF); role limitations due to physical health (RP); bodily pain (BP); social functioning (SF); vitality, energy, or fatigue (VT); general health perceptions $(\mathrm{GH})$; role limitations due to emotional problems (RE); general mental health $(\mathrm{MH})$; physical component score (PCS); and mental component score (MCS). Horizontal lines within boxes, boxes, error bars represent median, interquartile range, and range, respectively

\section{Discussion}

The management of patients with limb length discrepancies or segmental bone defects following fractures of the lower limb is complicated due to the protracted postoperative period of external fixation. In the three patients with segmental transport, the fixators were in place for almost 14 months; the distraction periods alone were 8 months for the femur and 5 months for the tibia. On average, it takes approximately 1.5 months to grow $1 \mathrm{~cm}$ of consolidated bone. This growth rate is in accordance with the published literature [3]. During this prolonged period of external fixation, the patient is at risk of developing pin tract infections. In this case series, eight out of fifteen patients developed a pin tract infection of which four patients underwent operative debridement. The prevalence of pin tract infections in literature has been reported to be as high as $95 \%[3,10]$. Furthermore, in the lengthening group, two patients underwent an additional operation, one for premature consolidation and another had a talocrural arthrodesis due to intractable ankle pain. All segmental transport patients underwent an additional operation for plate fixation and autogenous bone grafting to allow consolidation at the docking site.

The purpose of this study was to assess the long-term quality of life and functional outcome following distraction osteogenesis of the lower limb in cases of post-traumatic leg length discrepancy and bone defects. After a mean follow-up of 9 years, the quality of life and lower limb function were assessed with SF-36 and LEFS questionnaires.

The scores in the domains of physical functioning and bodily pain were slightly lower when compared with the general population. The scores were two points lower for physical functioning and one point for bodily pain when compared to the SF 36 norm-based scores for the US population. In all other six domains, the patients' scores were comparable with the general population.

The results of the LEFS questionnaire showed overall function of the lower extremity to be in the 'moderate difficulty' class for patients who underwent segmental transport and in 'a little bit of difficulty' for those who underwent lengthening. The mean mechanical axis deviation measured was $21 \mathrm{~mm}$ with five patients showing varus and eight patients showing valgus malalignment.

The limitation in interpreting these results is the sample group which is a case series. In general, the young patients as studied here are extremely motivated and cooperative. There is a selection bias because operative treatment was allocated by the treating surgeon. This may explain the quality of life scores that are equivalent compared with the general population in each domain. However, what this case series does show is that the quality of life in the long run is not influenced by prolonged external fixator use and the complications of pin tract infections or secondary operative interventions in these motivated, young and cooperative patients. The same is true for function of the 
lower extremity; these results may be influenced by coping behavior because of the long follow-up. These results indicate these patients, despite the severity of the initial injuries and prolonged treatment, have little or only moderate difficulties in daily activities including hobbies.

The mean MAD of $21 \mathrm{~mm}$ appeared not to influence the quality of life or functional outcome scores despite the mean follow-up of 9 years. However, longer follow-up may possibly show a higher rate of degenerative arthritis of the knee and ankle joint. Theoretically, distraction for lengthening or segmental transport is directed in line with the mechanical axis. Application of a monolateral fixator may introduce, in addition to the distraction vector, a varus or valgus directive force. This especially applies when the monolateral fixator is not exactly parallel to the mechanical axis. Paley described the problem of axis deviation when using a ring fixator, due to imbalance between muscle forces on different sides of the bone. $\mathrm{He}$ found that osteotomies of the proximal femur tended to go into varus and procurvatum, whereas osteotomies of the distal femur tended to go into valgus and procurvatum. In comparison, osteotomies of the proximal tibia tended to go into valgus and procurvatum and the distal tibia into varus and procurvatum [2]. The distraction system used in this study did not allow for correction of mechanical axis deviation during distraction, but newer systems do offer this option. Recently, fixator-assisted lengthening over an intramedullary nail was described $[10,11]$. Kocaoglu et al. [11] concluded that the duration of external fixation was reduced compared with that required for classic treatment and the patients' comfort improved. In addition, the intramedullary nail prevented deformation of the regenerated bone. This technique of distraction osteogenesis may prevent mechanical axis deviation as well.

In this study, a subperiosteal corticotomy in the metaphyseal part of the bone was performed because of the greater blood flow and a thinner cortex which facilitates the corticotomy. Fischgrund et al. [12] reviewed 114 consecutive patients who underwent 140 lower extremity bone segment lengthening procedures using the Ilizarov external fixator. They found that diaphyseal lengthening healed more slowly than metaphyseal lengthening.

The duration of the latency period in this study was 7 days. The latency period in most published clinical studies has ranged from 3 to 10 days [3]. However, in an experimental canine model, the bone-healing index showed that new bone consolidation was best with zero-day latency in metaphyseal and diaphyseal lengthening [13].

The bone segments were distracted with a rate of one millimetre per day in four $0.25 \mathrm{~mm}$ increments. Experimental studies have demonstrated that rates ranging from 0.5 to $2 \mathrm{~mm}$ per day lead to bone formation after a metaphyseal corticotomy, but more than $2 \mathrm{~mm}$ per day may exceed the potential for vascular ingrowth at a diaphyseal site [3, 14].

One of the main limitations of the present study is its small sample size. This is due to the fact that the incidence of patients with post-traumatic limb length discrepancies or segmental defects from initial trauma or infection is relatively low. Only 15 patients were treated in 8 years in a level I trauma center in the Netherlands but, with improvements in reconstructive techniques, the indications for limb salvage and the number of patients needing this treatment may increase. Despite its small sample size, to our knowledge, this study is unique in regard to the duration and completeness of follow-up and assessment of quality of life and functional outcome.

The key finding of this study is that quality of life returned to a level comparable with the normal population after post-traumatic distraction osteogenesis. Functional outcome indicated 'moderate difficulty' in patients following segmental transport and 'a little bit of difficulty' following lengthening of healed fractures. These results of quality of life and function, taking into account the study limitations, may be extrapolated to young, motivated and cooperative patients with limb length discrepancy or bone defect problems after fractures.

\section{References}

1. Dahl MT, Gulli B, Berg T (1994) Complications of limb lengthening. A learning curve. Clin Orthop Relat Res 301:10-18

2. Paley D (1990) Problems, obstacles, and complications of limb lengthening by the Ilizarov technique. Clin Orthop Relat Res 250:81-104

3. Aronson J (1997) Limb-lengthening, skeletal reconstruction, and bone transport with the Ilizarov method. J Bone Joint Surg Am 79(8):1243-1258

4. Aronson J, Johnson E, Harp JH (1989) Local bone transportation for treatment of intercalary defects by the Ilizarov technique. Biomechanical and clinical considerations. Clin Orthop Relat Res 243:71-79

5. DeCoster TA et al (2004) Management of posttraumatic segmental bone defects. J Am Acad Orthop Surg 12(1):28-38

6. Green SA (1994) Skeletal defects. A comparison of bone grafting and bone transport for segmental skeletal defects. Clin Orthop Relat Res 301:111-117

7. Aaronson NK et al (1998) Translation, validation, and norming of the Dutch language version of the SF-36 health survey in community and chronic disease populations. J Clin Epidemiol 51(11):1055-1068

8. Binkley JM et al (1999) The Lower Extremity Functional Scale (LEFS): scale development, measurement properties, and clinical application North American orthopaedic rehabilitation research network. Phys Ther 79(4):371-383

9. Paley D, Tetsworth K (1992) Mechanical axis deviation of the lower limbs. Preoperative planning of multiapical frontal plane angular and bowing deformities of the femur and tibia. Clin Orthop Relat Res 280:65-71 
10. Zhang X et al (2007) Reconstruction with callus distraction for nonunion with bone loss and leg shortening caused by suppurative osteomyelitis of the femur. J Bone Joint Surg Br 89(11):1509-1514

11. Kocaoglu M et al (2009) Fixator-assisted acute femoral deformity correction and consecutive lengthening over an intramedullary nail. J Bone Joint Surg Am 91(1):152-159

12. Fischgrund J, Paley D, Suter C (1994) Variables affecting time to bone healing during limb lengthening. Clin Orthop Relat Res 301:31-37
13. Aronson J, Shen X (1994) Experimental healing of distraction osteogenesis comparing metaphyseal with diaphyseal sites. Clin Orthop Relat Res 301:25-30

14. Ganey TM et al (1994) Basement membrane of blood vessels during distraction osteogenesis. Clin Orthop Relat Res 301:132138 